\title{
First Steps in Eliminating the Need for Animals and Cadavers in Advanced Trauma Life Support ${ }^{\circledR}$
}

\author{
Christoph Kaufmann, MD, MPH, FACS \\ Scott Zakaluzny, BA \\ Alan Liu, PhD \\ Surgical Simulation Laboratory \\ National Capital Area Medical Simulation Center \\ Uniformed Services University of the Health Sciences
}

\begin{abstract}
The Advanced Trauma Life Support ${ }^{\circledR}$ course is designed to provide for optimal initial resuscitation of the seriously injured patient. The surgical skills component of this course requires the use of cadavers or anesthetized animals. Significant anatomical differences and ethical issues limit the utility of animals. The cost and difficulty in procuring cadavers makes widespread use of this option impractical. A combination of mannequin and computer-based surgical simulators is being developed to replace these animals and cadavers. The first of these simulators, a pericardiocentesis trainer, is complete.
\end{abstract}

\section{Introduction}

The Advanced Trauma Life Support course (ATLS ${ }^{\circledR}$ ) provides the doctor with effective strategies for initial management of the trauma patient. The ATLS ${ }^{\circledR}$ course is strongly recommended for all physicians and surgeons involved in the care of seriously injured patients. Globally, an average of 19,000 doctors are trained each year [1]. The entire ATLS $^{\circledR}$ course focuses on rapid assessment and treatment to afford the best possible patient outcome. The ATLS ${ }^{\mathbb{R}}$ course includes a surgical skills practicum in addition to skills stations teaching accurate patient assessment, triage, establishing and maintaining the airway, using imaging to identify various injuries, and trauma care decision making [1]. The procedures in the surgical skills portion of the course include: tube thoracostomy, surgical airway, pericardiocentesis, and diagnostic peritoneal lavage. Today, ATLS $^{\circledR}$ students learn these procedures on anesthetized animals or cadavers. Further consideration is needed since anesthetized animals do not present the correct anatomy for realistic training, are not reusable, are expensive, and raise ethical issues. Cadavers, which have the correct anatomy, still pose problems because they are expensive and can be difficult to procure. Tissue degradation and frozen cadaver specimens present additional problems.

Recent advances in computer simulation technology permit many of these procedures to be practiced in a virtual environment. In this paper, we provide introduction to our work in developing an ATLS ${ }^{\circledR}$ surgical skills lab that eliminates the use of animals and cadavers. 


\section{Methods}

The National Capital Area Medical Simulation Center is a state-of-the art medical education facility at the Uniformed Services University of the Health Sciences (USUHS). It consists of three functional areas: clinical examination rooms, computer lab, and surgical simulation lab. USUHS has received permission from the American College of Surgeons to examine the utility of using computer-based surgical simulators in teaching the ATLS ${ }^{\circledR}$ surgical skills practicum. The surgery simulation lab includes the MedSim patient simulator [2], a desktop 3D haptic reach in virtual environment, a BDI vascular anastomosis simulator, CathSim IV insertion simulators, Ultrasim ultrasound simulator, HT Medical PreOp broncoscopy simulator, and other simulation technologies.

The MedSim patient simulator is a computer-controlled mannequin designed to simulate a human patient. Among the various features on this device are reactive pupils, arm motion response to painful stimulus, and both radial and carotid pulses. Two ATLS ${ }^{\circledR}$ surgical skills can be taught on the simulator. The simulator can accept and respond to chest tubes used in tube thoracostomy. The simulator also permits the creation of a surgical airway and responds with the appropriate physiological reaction to improved ventilation and oxygenation. The mannequin's airway is connected to a computer which analyzes the percent oxygen in inhaled air, displays blood oxygen saturation results on a pulse-oxygen monitor, and exhales carbon dioxide. The mannequin allows for the delivery of and detection of eighty-five drugs. This is a very abbreviated list of features of this highly useful teaching tool. The simulator can reproduce physiologic responses consistent with various forms of injury by using the computer interface to control forty different patient attributes from age to insulin production and invoke up to thirty medical complications.

One of the conditions which the mannequin can simulate is cardiac tamponade, a diagnosis which mandates pericardiocentesis, although this procedure cannot be performed on the MedSim mannequin. Pericardiocentesis is a simple needle-based procedure and lends itself to virtual reality simulation. Pericardiocentesis involves the needle aspiration of excess fluid (e.g., blood) from the pericardial sac to permit the heart to fill and function properly. Since this is an emergency procedure, external guidance (e.g., fluoroscopy) is not available. The physician must be familiar with acceptable entry locations and angles. The physician must also know how far to advance the needle.

We have developed a virtual reality based simulator for pericardiocentesis. The simulation is based on a desktop 3D virtual environment similar to that described in [3]. A flat panel liquid crystal display (LCD) is used to generate stereoscopic images of the environment. The LCD display incorporates a grid to focus light rays from alternate pixels to each eye, permitting a different view to be generated for each eye generating the 3D perspective [4]. Special eyewear is not required. This display facilitates a more comfortable viewing of the virtual environment over extended periods of time. 
The pericardiocentesis simulator is running on a PC with a $550 \mathrm{MHz}$ Intel Pentium III Xeon ${ }^{\circledR}$ processor, a half a gigabyte of memory, and an Evans and Sutherland Tornado $3000^{\mathbb{R}}$ video card. The image on the flat screen LCD is reversed so that it can be viewed in a mirror situated horizontally below and in front of the monitor. (Fig. 1) The student looks down at the horizontally situated mirror and sees a 3-D rendered field with an external view of a virtual thorax and a virtual cannula. A Phantom haptic interface [5] controls the position and orientation (pose) of this virtual cannula. In order to increase the realistic feel of the simulation, the Phantom is set-up in such a way that the arm extends underneath the viewing mirror so that the stylus on the Phantom is moving in three-space with the same orientation and position as the cannula it represents in the virtual image being viewed. (Fig. 2) This preserves the hand eye axis of the operator. Using the Phantom, the student selects an entry location and angle, then inserts the cannula into the chest. (Fig. 3) Haptic feedback is provided to simulate the effect of both an increase and a decrease in resistance associated with that of the cannula meeting and penetrating the skin and chest wall. The program provides both positive and negative feedback by having the cannula fill with blood from the pericardium and by displaying a warning message, respectively. (Fig. 4 \&5) If the student chooses either the wrong entry point and/or advances the needle in the wrong direction an obvious warning message appears (allowing for correction).

\section{Discussion}

Medical simulators provide significant advantages over animals and cadavers. Simulators generate repeatable scenarios that are impossible to predictably simulate with cadavers or animals. Not only can difficult situations be simulated, they can be repeated as many times as needed since no specimen is destroyed. By using the same simulator and same loaded scenario, the computer simulation provides a means of ensuring uniformity in training and evaluating performance. In addition, rare anatomic variations or pathology can be easily emulated. These provide exposure to unusual cases hands-on as contrasted with a description in a book or lecture. The program's warnings also act as an additional way of instructing students. The warnings provide the immediate feedback that allows the student to correct their actions and learn to do the procedure properly. The obvious advantage of simulators is that they are reusable and, following initial start-up cost, they require only minimal maintenance compared to purchasing new cadavers or animals. This makes simulators more cost-effective over the long term. Most importantly, no ethical issues are involved when using computer-based simulation.

Computer-based and mechanical medical simulators do have disadvantages. Limited visual and tactile realism can still be a problem (e.g., bleeding is difficult to simulate). Simulators require high-end equipment and new programming which can create a high initial cost. In addition, the field of medical simulators is still in its infancy and is pushing the envelope of the present technology. Many useful procedures currently do not have adequate simulations because the technology does not exist to support 
Figure 1- Demonstrates the flat screen LCD and mirror used to view the 3D rendered field.

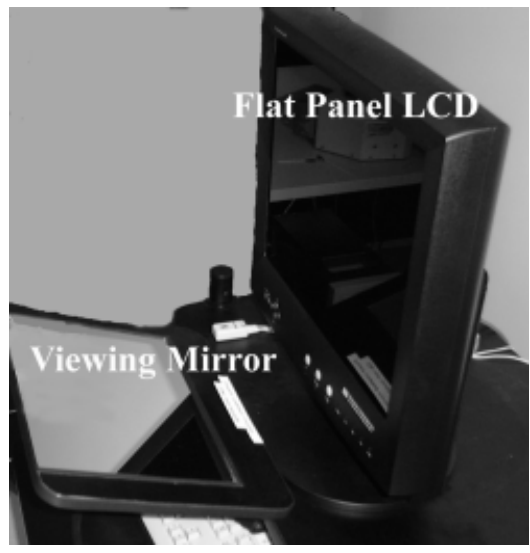

Figure 2- Demonstrates the flat screen LCD, mirror, and Phantom haptic feedback device.

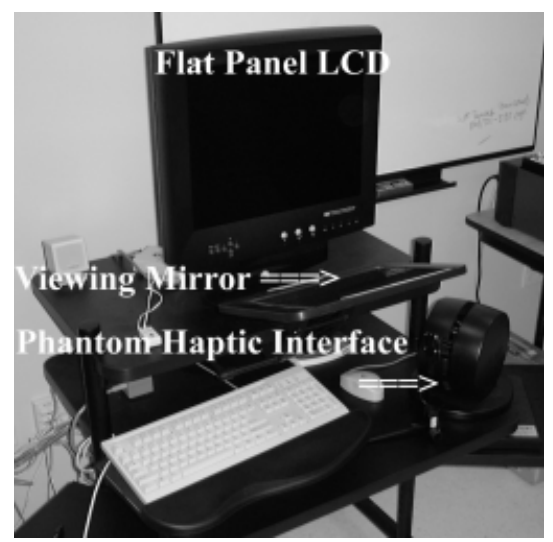

Figure 3- Screen capture demonstrates the field of view and the cannula. Viewed here in 2D and black and white as opposed to $3 \mathrm{D}$ and color.

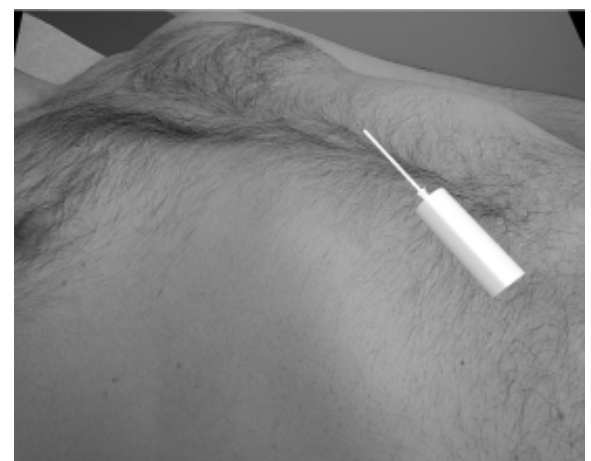


Figure 4- Screen capture demonstrates the cannula filling with blood in a successful procedure.

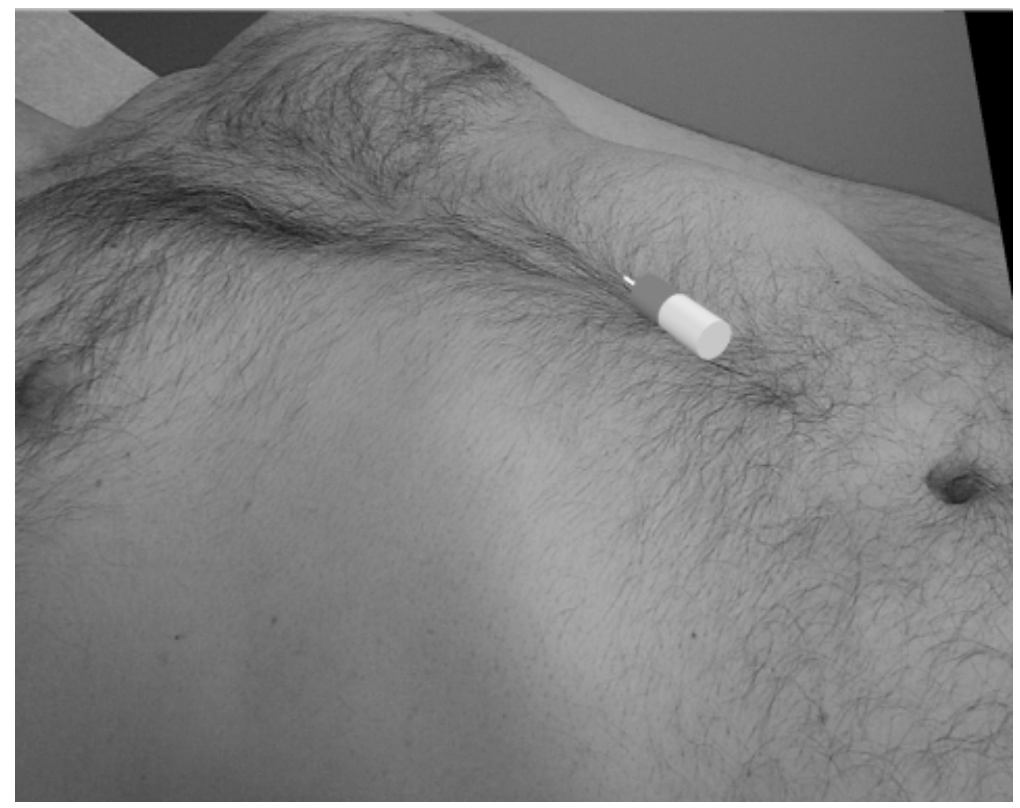

Figure 5- Screen capture demonstrates example of a warning message.

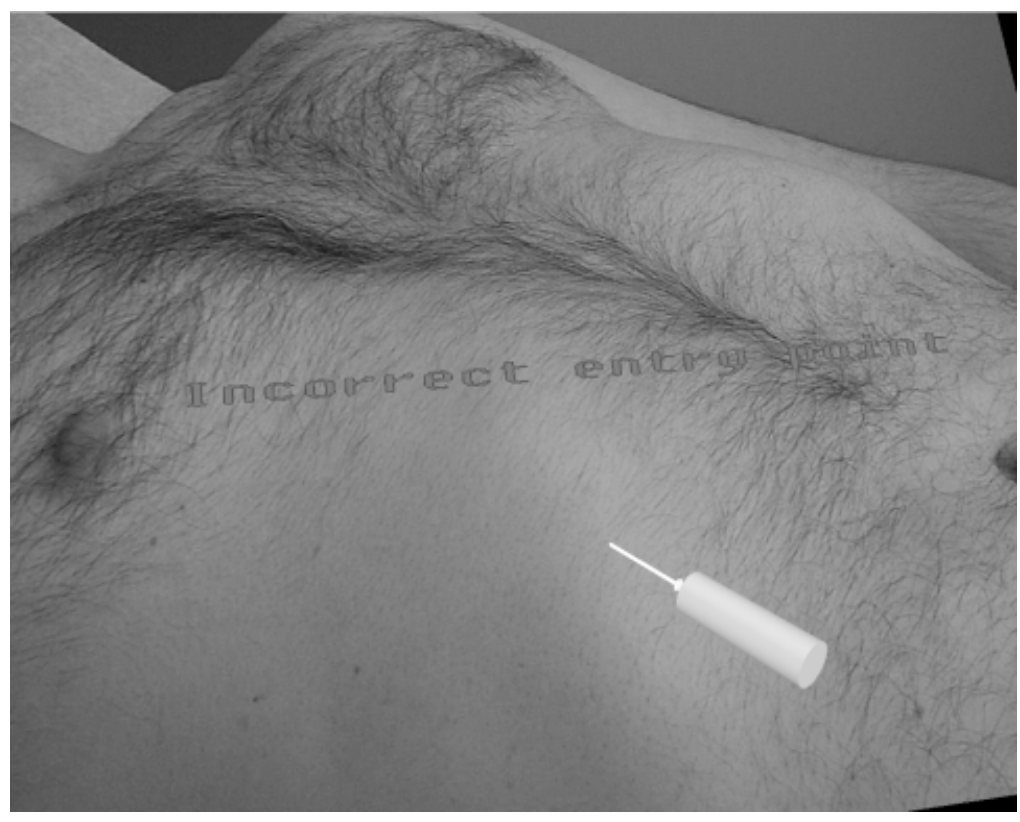


them. The simulators that are being developed now are used to assure that specific tasks can be completed properly, but many other surgical procedures cannot be simulated as of yet. This is primarily because of the complexity of human anatomy, the need to represent physiologic processes such as bleeding, and the lack of accurate haptic representation of various human tissues. [6]

\section{Conclusion}

Computer-based medical simulators are a viable alternative to animals and cadavers for teaching ATLS ${ }^{\circledR}$ surgical skills. A skill-specific surgical simulator has been developed for pericardiocentesis. The basic aspects of correct location, angle, and depth for cannula insertion can be demonstrated and evaluated. Work is on-going to evaluate the efficacy of this device and to develop a second surgical simulator, diagnostic peritoneal lavage.

Most recently, the pericardiocentesis simulator has been moved to a lower end platform that does not require the flat screen LCD or Phantom haptic device. This move was made to make the program more portable and fiscally feasible for widespread use. The development and testing of this system is on-going.

\section{References}

1. American College of Surgeons Committee on Trauma, "Advanced Trauma Life Support ${ }^{\mathbb{B}}$ for Doctors", $6^{\text {th }}$ edition, Chicago, IL (1997)

2. MedSim Advanced Medical Simulations, Ltd. Product literature. URL: www.medsim.com

3. Poston T., Serra L., "Dextrous virtual work", Communications of the ACM v. 39 (May) p. 37-45, 1996

4. Kaufmann, C., Liu A., Burris D., "DTI Autostereographic Display: Initial Evaluation," Medicine Meets Virtual Reality 2000, J.D. Westwood et al (Eds.) IOS Press, Amsterdam (2000) 156-158

5. Massie T.H., Salisbury J.K., "The PHANToM Haptic Interface: A Device for Probing Virtual Objects." Proceedings of the ASME Winter Annual Meeting, Symposium on Haptic Interfaces for Virtual Environment and Teleoperator Systems, Chicago, IL, Nov. 1994

6. Kaufmann, C., "Role of Surgical Simulators in Surgical Education," Asian J. Surgery 1999; 22 (4): 398-401 